\title{
The Role of Working Memory in Visual Selective Attention
}

\author{
Jan W. de Fockert,1* Geraint Rees,2 Christopher D. Frith,3Nilli Lavie1 \\ 1Department of Psychology, University College London, Gower Street, London WC1E 6BT, UK. 2Institute of \\ Cognitive Neuroscience, University College London, Alexandra House, 17 Queen Square, London WC1N \\ 3AR, UK. 3Wellcome Department of Cognitive Neurology, Institute of Neurology, 12 Queen Square, London \\ WC1N 3BG, UK.
}

*To whom correspondence should be addressed. Email: j.de-fockert@ucl.ac.uk

The hypothesis that working memory is crucial for reducing distraction by maintaining the prioritization of relevant information was tested in neuroimaging and psychological experiments with humans. Participants performed a selective attention task that required them to ignore distractor faces while holding in working memory a sequence of digits that were in the same order (low memory load) or a different order (high memory load) on every trial. Higher memory load, associated with increased prefrontal activity, resulted in greater interference effects on behavioral performance from the distractor faces, plus increased face-related activity in the visual cortex. These findings confirm a major role for working memory in the control of visual selective attention.

Despite a vast body of research on visual attention and on working memory, the interaction between the two has seldom been addressed. There have been a few recent suggestions that working memory may play a role in the control of selective attention $(1,2)$, but evidence for a specific role has been scarce. Here we show a direct causal role for working memory in the control of selective attention.

The most enduring issue in the study of attention is the extent to which distractor processing can be prevented (3). Lavie recently proposed that the level of perceptual load in a display is a crucial factor (4). Several studies have shown that distractors that could not be ignored in situations of low perceptual load (for example, when just a few task-relevant stimuli were presented) were successfully ignored in situations of high perceptual load (for example, when many relevant stimuli were present). Thus, less distrac- tor processing was found in the harder task.

Here we propose that the effect of task difficulty on distractor processing depends critically on the mental process that is loaded. We suggest that directing attention appropriately requires the active maintenance of stimulus priorities in working memory, specifying which stimuli are currently relevant. A high load on working memory should therefore lead to reduced differentiation between high- and low-priority stimuli (that is, between targets versus distractors in a selective attention task). This leads to the counterintuitive prediction of an opposite effect to that found for perceptual load: Higher working memory load should increase distractor processing. 


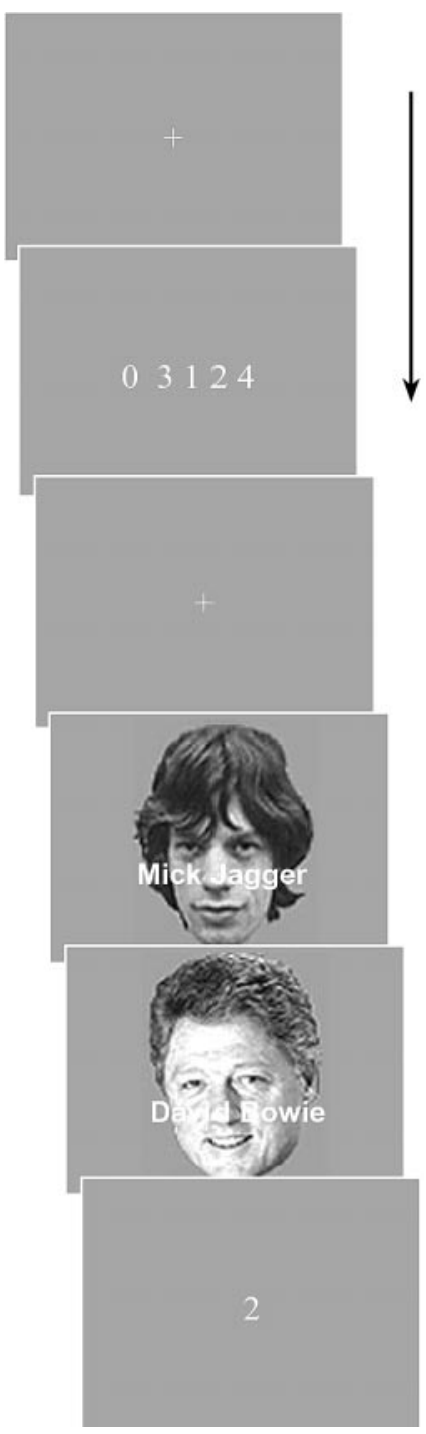

Fig. 1. Example of a high working memory load trial with two attention displays. After a 500-ms fixation display, the memory set for that trial was presented for $1500 \mathrm{~ms}$. Under low working memory load, the digits were always in the following order: $0,1,2,3,4$. After the memory set, a fixation display was presented for $850 \mathrm{~ms}$, followed by two, three, or four attention displays. The number of attention displays was varied in order to make the onset of the memory probe unpredictable, thus ensuring that the current memory set was actively rehearsed throughout the trial. Each attention display was presented for $500 \mathrm{~ms}$ and was followed by a 1250-ms blank response interval. After the final attention display, a memory probe was presented for 3000 ms. Participants were requested to report the digit that followed this probe in the memory set (to press "4" in this example). In order to ensure that all four responses (including " 1 " in low working memory load trials) were used, we presented a "o" before the four-digit memory set. Thus, the correct response to memory probe "o" would have been " 3 " in this example.

To test this hypothesis, we combined two unrelated tasks-one requiring visual selective attention and the other working memory - with the prediction that increasing load in the working memory task should increase the processing of visual distractors in the selective attention task. Even though the working memory and visual attention tasks were unrelated (Fig. 1), high load in the working memory task should reduce the availability of working memory for maintaining stimulus priorities in the selective attention task and thus lead to greater intrusion of irrelevant distractors.

We first tested this prediction in a behavioral experiment with 10 participants (Fig. 1). In the selective attention task, observers were asked to classify famous written names as pop stars or politicians while ignoring distractor faces (5). The distractor faces were equally likely to be congruent with the target name, incongruent with the target name, or anonymous. Distractor face processing was assessed by comparing classification reaction times (RTs) between the congruent and incongruent conditions. This task was interleaved with a working memory task for digit order. Load was manipulated in the memory task by requiring participants to remember either a fixed order of digits or a different order of digits on each trial (6). Manipulation of working memory load was effective. RTs to the memory probe increased from 953 to $1394 \mathrm{~ms}$ between low and high memory load. More important, in the selective attention task, there was a significant interaction between distractor condition and 
working memory load [F(1,9) 5 13.36, P , o.01]; distractor interference effects were significantly greater during high $(78 \mathrm{~ms})$ than low $(46 \mathrm{~ms})$ working memory load, indicating more distractor processing in the selective attention task with high load in the working memory task (7).

Table 1. Brain regions showing significant activation related to working memory load (high load. low load). Shown are voxels in which activity was greater than $\mathrm{P}<0.05$, corrected for multiple comparisons.

\begin{tabular}{|c|c|c|c|c|}
\hline \multirow[t]{2}{*}{ Cortical area } & \multicolumn{3}{|c|}{ Talairach coordinates } & \multirow{2}{*}{$\begin{array}{c}\mathrm{t} \\
\text { value }\end{array}$} \\
\hline & $\mathrm{x}$ & $\mathrm{y}$ & $\mathrm{z}$ & \\
\hline \multicolumn{5}{|c|}{ Frontal areas } \\
\hline Precentral gyrus (BA 4) & -50 & -8 & 46 & 8.71 \\
\hline Precentral gyrus & 52 & 0 & 50 & 6.76 \\
\hline (BA 6) & 26 & -10 & 50 & 5.10 \\
\hline Inferior frontal gyrus & -54 & 8 & 20 & 7.87 \\
\hline$($ BA 44) & -42 & 2 & 32 & 6.23 \\
\hline Medial frontal gyrus (BA 6) & -4 & 4 & 60 & 6.45 \\
\hline Middle frontal gyrus & -24 & 4 & 58 & 5.45 \\
\hline$(\mathrm{BA} 6)$ & -34 & -4 & 56 & 4.94 \\
\hline Insula & 44 & 14 & 22 & 5.43 \\
\hline \multicolumn{5}{|c|}{ Other areas } \\
\hline $\begin{array}{l}\text { Superior temporal gyrus (BA } \\
22 \text { ) }\end{array}$ & -56 & 8 & 2 & 5.58 \\
\hline Precuneus (BA 7) & -20 & -62 & 52 & 5.41 \\
\hline Superior parietal lobe (BA 7) & 36 & -52 & 54 & 5.01 \\
\hline
\end{tabular}

These findings provide preliminary support for our hypothesis that the availability of working memory for actively maintaining stimulus-processing priorities is crucial for directing attention to relevant rather than irrelevant stimuli, and thus minimizing the intrusion of irrelevant distractors. Hence, more distractor processing is found under high working memory load [this is the opposite pattern to that found for perceptual load $(4,8-10)]$. We next investigated neural responses to the distractors. If the rejection of irrelevant visual distractors in a selective attention task depends on the availability of working memory to sustain stimulus priorities for attention, then greater activity related to the distractor faces should be found in the visual cortex under conditions of higher working memory load.

Functional magnetic resonance imaging (fMRI) was used to measure brain activity while participants performed the interleaved attention and memory tasks. To measure distractorrelated activity in the selective attention task, we included conditions in which the distractor face was absent and compared neural activity in these conditions to conditions in which the distractor face was present (11). Behavioral data from the memory task confirmed that our manipulation of working memory load was effective (RTs to the memory probe were $1400 \mathrm{~ms}$ with high memory load compared to $921 \mathrm{~ms}$ with low working memory load). RTs in the selective attention task revealed that interference from incongruent (versus congruent) distractor faces was again greater with high working memory load $(73 \mathrm{~ms})$ than with low working memory load (32 ms) (12).

Group analysis of the fMRI scans from six participants (13) revealed several areas in the frontal cortex where activity was greater during conditions of high than of low working memory load (Fig. 2 and Table 1). These areas included the inferior frontal gyrus (BA 44), the middle frontal gyrus (BA 6), and the precentral gyrus (BA 4), which have all been previously associated with working memory load (14-17). These findings confirm the validity of our task for manipulating load in working memory. 


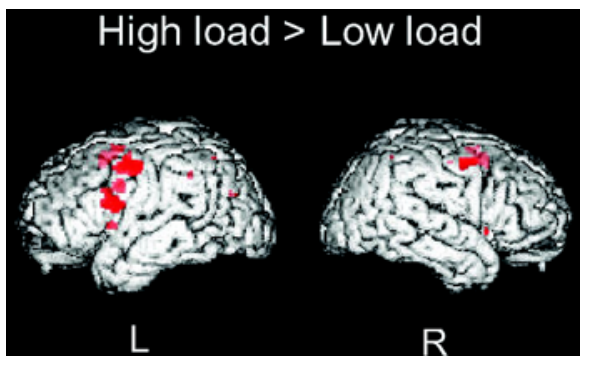

Fig. 2. Activity related to working memory load. Shown are left and right lateral views of a T1-weighted anatomical template image in Talairach space (27), on which are superimposed loci where activity was significantly greater $(\mathrm{P}$, 0.05, corrected for multiple comparisons) during high working memory load than during low working memory load.

Neural activity related to the presence (versus absence) of distractor faces in the selective attention task was modulated by working memory load. Activity related to the presence of distractor faces in the fusiform gyrus and extrastriate visual cortex was significantly greater under conditions of high than of low working memory load (Fig. 3A). Statistically, this finding is reflected in the interaction term of our factorial design (memory load crossed with the presence of a distractor face). Three sites showed such a significant interaction: the bilateral fusiform gyri, right inferior occipital lobe, and left lingual gyrus (see Table 2 for coordinates and $t$ values). These areas have been associated with face processing in earlier studies (18-21), and they all showed greater distractorrelated activity under conditions of high working memory load than under low load (Fig. 3B). These findings imply that the processing of distractor faces, presented in the selective attention task, was more extensive under high working memory load than low working memory load in a concurrent memory task.

Taken together, our behavioral and functional imaging results demonstrate an interaction between working memory and selective attention. High working memory load resulted in increased interference effects on performance from distractor faces and also in a significant increase of activity elicited by distractor faces in visual areas known to be selective for face processing. Even though working memory and selective attention were manipulated in two separate and unrelated tasks, they interacted in the very specific sense predicted by our hypothesis. These results provide evidence for the theoretical suggestion that working memory serves to control visual selective attention in the normal human brain.

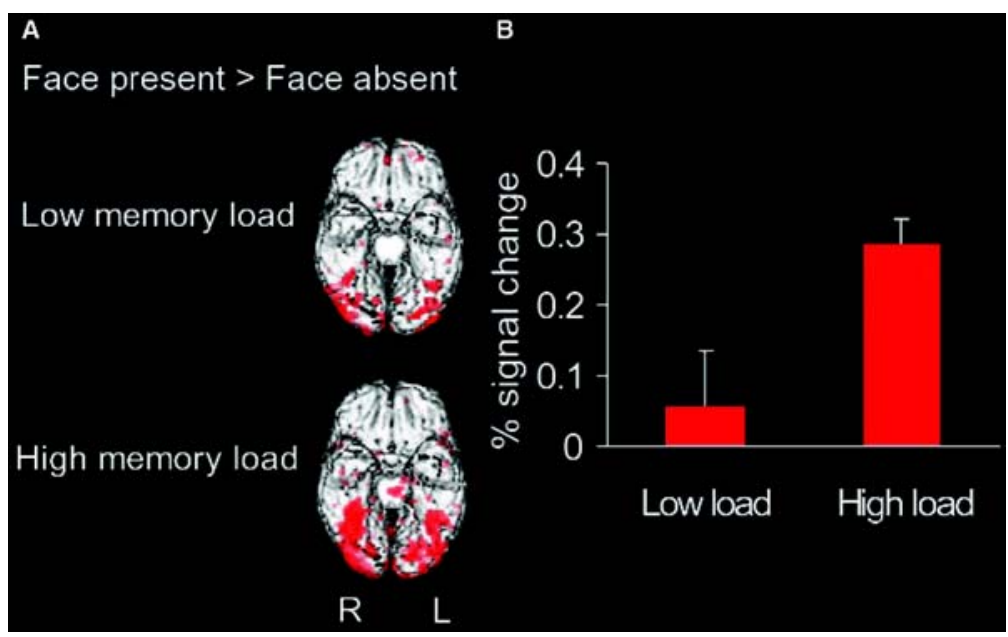

Fig. 3. Distractor-related activity in high versus low working memory. (A) Two views of the ventral surface of the template brain used in Fig. 2, on which are superimposed loci where activity was significantly greater in the presence than in the absence of distractor faces under conditions of low working memory load (top) and high working memory load (bottom). A threshold of $\mathrm{Z} 53.10$ (corresponding to $\mathrm{P}, 0.001$, uncorrected) is used for display purposes. (B) Mean distractor-related activity ( percent signal change for face presence minus face absence) for the maxima of the interaction in the right fusiform gyrus $(36,-64,-16)$, plotted separately for low and high working memory load. Data are averaged across participants. Error bars represent interparticipant standard error. 
Table 2. Brain regions showing significant activation related to the interaction of working memory load and the presence of distractor faces. In these areas, activity related to distractor face presence (versus absence) was greater under conditions of high working memory load than under low working memory load. Shown are voxels in which activity was greater than P , 0.001, uncorrected [because of our prior anatomical hypothesis about these face-related areas (18-20)]. Outside facerelated areas, no significant activity was seen above a threshold of $\mathrm{P}, 0.05$, corrected for multiple comparisons.

\begin{tabular}{lcccc}
\hline Cortical area & \multicolumn{3}{c}{ Talairach coordinates } & $\mathrm{t}$ \\
\cline { 2 - 4 } & $\mathrm{x}$ & $\mathrm{y}$ & $\mathrm{t}$ & $\mathrm{t}$ \\
\hline & & & -16 & 3.64 \\
Fusiform gyrus & 36 & -64 & -24 & 3.63 \\
(BA 19/37) & 40 & -38 & -16 & 3.28 \\
& 48 & -60 & -16 & 3.10 \\
Inferior occipital gyrus & -48 & -54 & 0 & 3.42 \\
(BA 18) & 38 & -90 & -6 & \\
Lingual gyrus (BA 18) & -16 & -78 & & \\
\hline
\end{tabular}

Our imaging finding that effects of working memory load in the prefrontal cortex interact with distractor-related activity in posterior cortices supports the notion that the frontal lobes play an important role in the control of attention $(22,23)$. We have shown that frontal control of attention by working memory is a critical factor in distractor processing, a notion that has been alluded to speculatively in some theories of attention $(1,2)$ and is consistent with neuropsychological reports of deficits in selective attention after frontal lobe damage (22), as well as with recent electrophysiological findings indicating the maintenance of task-relevant information in monkey prefrontal neurons (24). The present results, when taken together with the recent reports on the role of perceptual load in selective attention $(4,8-10)$, help in solving the long-standing controversy regarding the extent of processing of irrelevant distractors. Although distractors are usually perceived to some extent in situations of low perceptual load (as when just a single target name and a single distractor were presented), the extent to which the distractors intrude is crucially determined by the availability of working memory.

\section{References and Notes}

1. A. Baddeley, Q. J. Exp. Psychol. 49A, 5 (1996).

2. R. Desimone, J. Duncan, Annu. Rev. Neurosci. 18, 193 (1995).

3. Throughout four decades of debate, there have been many demonstrations of attentional modulation of distractor perception (25). However, many other studies have demonstrated that irrelevant distractors can sometimes be perceived (26), thus fueling an enduring controversy.

4. N. Lavie, J. Exp. Psychol. Hum. Percept. Perform. 21, 451 (1995).

5. A. W. Young, A. W. Ellis, B. M. Flude, K. H. McWeeny, D. C. Hey, J. Exp. Psychol. Hum. Percept. Perform. 12, 466 (1986).

6. Each digit in the memory task subtended $0.4^{\circ}$ of visual angle horizontally and $0.6^{\circ}$ vertically. The entire memory set subtended $3.4^{\circ}$ horizontally. The names for the attention task subtended between $3.9^{\circ}$ and $5.3^{\circ}$ horizontally and $0.6^{\circ}$ vertically. Distractor faces subtended approximately $5.0^{\circ}$ horizontally and $6.0^{\circ}$ vertically (see Fig. 1 for timing details). In the behavioral study, viewing distance was approximately $60 \mathrm{~cm}$, and responses to both tasks were collected from the numeric keypad on the computer keyboard.

7. Error rates showed a pattern similar to that of the RT data. Error rates in the memory task increased from $4 \%$ under low working memory load to $8 \%$ under high working memory load. In the selective attention task, distractor congruency effects increased from $3 \%$ under low working memory load to $4 \%$ under high working memory load. Overall RTs and error rates in the selective attention task were not affected by working memory load. Average overall RT ( pooled across distractor conditions) was $739 \mathrm{ms,}$ 
error rate $5 \%$, under low working memory load, and $752 \mathrm{~ms}$, error rate $5.5 \%$, under high working memory load.

8. G. Rees, C. D. Frith, N. Lavie, Science 278, 1616 (1997).

9. N. Lavie, S. Cox, Psychol. Sci. 8, 395 (1997). 10. N. Lavie, E. Fox, J. Exp. Psychol. Hum. Percept. Perform. 26, 1038 (2000).

11. Informed consent was obtained from six participants (two males and four females). The mean age was 24.8 years (age range, 22 to 29 years). All participants were right-handed and were native English speakers. A 2T Siemens VISION system was used to acquire blood oxygenation level-dependent contrast image volumes. Each image volume comprised 32 contiguous $3-\mathrm{mm}$ axial slices, with an in-plane resolution of $3 \mathrm{~mm}$ by $3 \mathrm{~mm}$ positioned to include both the frontal and posterior temporal cortex. Volumes were acquired continuously every $2800 \mathrm{~ms}$ (or $3170 \mathrm{~ms}$ for participant 1 ). A total of 720 functional volumes were acquired in six separate runs for each participant. Each run consisted of four epochs: one epoch for each experimental condition from the 2 (working memory load: high versus low) 32 (distractor condition: face present versus face absent) factorial design, and the order of presentation was counterbalanced. The experimental epochs had a fixed duration consisting of 20 imaging volumes each and were followed by a 10-volume fixation epoch. Each epoch had five memory trials, and the total number of attention displays per epoch was always 15 (either one trial with two attention displays, three trials with three displays, and one trial with four displays; or two trials with two attention displays, one trial with three displays, and two trials with four displays).

12. Again, error rates showed a pattern that was similar to that of the RT data. Error rates in the memory task increased from $3 \%$ under low working memory load to $9 \%$ under high working memory load. In the selective attention task, distractor congruency effects increased from $1 \%$ under low working memory load to $5 \%$ under high working memory load. Overall RTs and error rates in the selective attention task were not affected by working memory load. Average overall RT ( pooled across distractor conditions) was $741 \mathrm{~ms}$, error rate $4.8 \%$, under low working memory load, and $746 \mathrm{~ms}$, error rate $4.4 \%$, under high working memory load.

13. Data were analyzed using a fixed effect model in SPM99 (www.fil.ion.ucl.ac.uk/spm). After the first six scans of each run were discarded to allow for T1 equilibration effects, the imaging series was realigned, spatially normalized to Talairach space (27), and smoothed with an isotropic 6-mm Gaussian kernel (28). Voxels that were activated during the experimental conditions were identified with a statistical model containing delayed boxcar waveforms that represented the mean activity evoked in each condition. In addition, high pass filtering removed participant- specific low frequency drifts in signal, and global changes in activity were removed by proportional scaling. Each component of the model served as a regressor in a multiple regression analysis (29). Linear contrasts between the experimental conditions were used to test the hypotheses of interest, creating a statistical parametric map of the resultant $t$ values. Resultant regions of activation were characterized in terms of their peak height.

14. J. D. Cohen et al., Nature 386, 604 (1997).

15. E. E. Smith, J. Jonides, Cognit. Psychol. 33, 5 (1997).

16. S. M. Courtney, L. G. Ungerleider, K. Keil, J. V. Haxby, Nature 386, 608 (1997).

17. S. M. Courtney, L. Petit, J. M. Maisog, L. G. Ungerleider, J. V. Haxby, Science 279, 1347 (1998).

18. N. Kanwisher, J. McDermott, M. M. Chun, J. Neurosci. 17, 4302 (1997).

19. M. L. Gorno Tempini et al., Brain 121, 2103 (1998). 20. I. Gauthier, P. Skudlarski, J. C. Gore, A. W. Anderson, Nature Neurosci. 3, 191 (2000).

21. The distractor activity by working memory load interaction was more apparent in righthemisphere regions than in the left hemisphere ( Table 2). This is consistent with our suggestion that working memory load results in increased activity specifi- cally related to face distractors [see previous reports of right-hemisphere dominance for face processing (18-20)].

22. T. Shallice, P. Burgess, in Frontal Lobe Function and Dysfunction, H. Levin, A. J. Benton, Eds. (Oxford Univ. Press, New York, 1991). 
23. M. I. Posner, S. E. Petersen, Annu. Rev. Neurosci. 13, 25 (1990).

24. G. Rainer, W. F. Asaad, E. K. Miller, Nature 393, 577 (1998).

25. S. Yantis, J. C. Johnston, J. Exp. Psychol. Hum. Percept. Perform. 16, 135 (1990).

26. S. P. Tipper, Q. J. Exp. Psychol. 37A, 571 (1985).

27. J. Talairach, P. Tournoux, Co-Planar Stereotaxic Atlas of the Human Brain, M. Rayport, Transl. ( Thieme, New York, 1988).

28. K. J. Friston et al., Hum. Brain Mapp. 3, 165 (1995a).

29. K. J. Friston et al., Hum. Brain Mapp. 2, 189 (1995b).

30. Supported by the Wellcome Trust (G.R. and C.F.) and by a Medical Research Council (UK) career award and a Biotechnology and Biological Sciences Research Council grant to N.L. 